\title{
Die Temperaturempfindlichkeit isolierter Lorenzinischer Ampullen
}

\author{
Friedrich-Karl Pierau und Hans D. Meissner \\ William-G.-Kerckhoff-Herzforschungsinstitut der Max-Planck-Gesellschaft, \\ Bad Naubeim
}

\begin{abstract}
The thermal sensitivity of isolated ampullae of Lorenzini. The ampullae of LORENZINI of elasmobranchs have a high sensitivity to temperature, as has been found by SAND (1938) and Hensel (1955) in single fibre preparations. Rapid cooling results in a steep, although temporary increase in impulse frequency; rapid warming is followed by a short and temporary inhibition of discharges. This behaviour very closely resembles that of the "cold" receptors of warm blooded animals. During experiments with isolated ampullae of LoRENZINI of the infra- and supraorbital group of Scyllium canicula, we found in two animals some ampullae with an opposite response to temperature changes: rapid cooling inhibited the discharges of the ampullae, while rapid changes to warmth initiated a temporary increase in impulse rate. In some of the ampullae a kind of "adaptation" after fast temperature changes was observed which was also different from what has been described by other authors. The high dynamic response after rapid cooling was followed by a strong diminution in discharge rate. Only after several minutes the impulse frequency again rose to a new and constant level. With rapid warming the behaviour of the spike rate was the mirror image: initially, the frequency dropped to low values but then rose again to a level which was higher than before warming. After this maximum the discharge role fell to a constant frequency. From the results it may be concluded that the localization of the ampullae might be of importance for the "specific" function of the receptor as well as for the differentiation of the peripheral stimulus pattern.
\end{abstract}

\section{EINLEITUNG}

Seit den Versuchen von SAND (1938) ist bekannt, daß Lorenzinische Ampullen von Selachiern eine hohe Empfindlichkeit gegenüber thermischen Reizen haben. In eingehenden Untersuchungen hat HENSEL (1955) die Erregungsgesetze der Lorenzinischen Ampullen unter verschiedenen Temperaturbedingungen dargelegt und auf die große Übereinstimmung mit dem Entladungsmuster der Kaltrezeptoren bei Warmblütern hingewiesen. Ein Verhalten, wie Dod't \& ZotTerman (1952) es für die Warmrezeptoren bei Warmblitern beschrieben haben, konnte er in seinen Versuchen nicht beobachten. Inzwischen wurde von Hensel (1957), Murray $(1955,1960,1962)$ und LoEWENSTEIN $(1960,1962)$ eine Sensitivität der Lorenzinischen Ampullen gegenüber mechanischen, chemischen und elektrischen Reizen festgestellt, wobei die bemerkenswert große Empfindlichkeit gegenüber Temperaturänderungen unbestritten blieb. 
Uber die biologische Bedeutung dieser in so großer Anzahl bei den Elasmobranchiern vorkommenden Organe ist bisher noch keine Entscheidung gefallen. Als Argument gegen ihre Funktion als Thermorezeptoren werden der anatomische Bau und die relativ große Entfernung der eigentlichen Sinnesorgane von der Körperoberfläche angeführt. Für die Perzeption von elektrischen und chemischen Reizen scheint es dagegen einen gemeinsamen elektrochemischen Mechanismus der Reizübertragung von der Ampullenöffnung zu den Rezeptoren mit relativ kurzer Zeitkonstante zu geben (LOEWENSTEIN \& IsHixo 1962). Verhaltensphysiologische Versuche von DijkgRAAF (1963) haben die Ansprechbarkeit der Tiere auf künstliche elektrische Reize von $3, \mu \mathrm{V} / \mathrm{cm}$ ergeben, was den elektrophysiologischen Ergebnissen an isolierten Lorenzinischen Ampullen entspricht. Nach Ausschaltung der Lorenzinischen Ampullen konnte DijKgraAp mit $2 \mathrm{mV} / \mathrm{cm}$ keine Reaktion mehr auslösen.

In unseren Untersuchungen über Stoff wechselvorgänge an Thermorezeptoren, bei denen uns die Lorenzinischen Ampullen als Modell dienten, erhielten wir auch einige elektrophysiologische Daten, die eine Ergänzung zu den Befunden von SAND und HeNSEL darstellen.

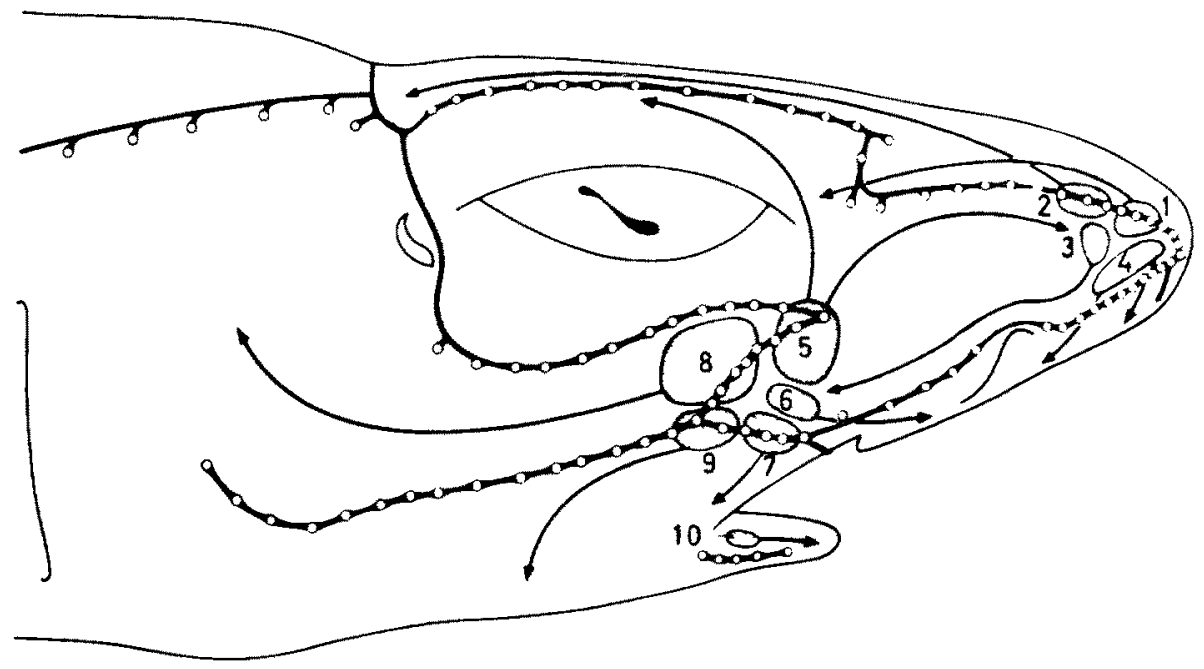

Abb. 1: Gruppen von Endampullen am Kopf des Katzenhaies, Scyllizm canicula, von rechts gesehen; 1-4: supraorbitale (= rostrale) Ampullengruppen; 5-9: infraorbitale Ampullengruppen; 10: mandibuläre Ampullengruppe; die Pfeile geben die Hauptverlaufsrichtung der jeweils zugehörigen Gallertröhren an. (Nach DijkgRaAf \&L KaLmuN 1963)

\section{MATERIAL UND METHODIK}

Die Versuche wurden in der Meeresstation der Biologischen Anstalt Helgoland in den Monaten März bis Mai an 24 Exemplaren von Scyllium canicula durchgeführt. Die Katzenhaie stammten aus dem Armelkanal und wurden seit Ende Januar bei ca. $15^{\circ} \mathrm{C}$ gehältert. Zur Präparation von Einzelampullen wurden die infraorbitalen und in einigen Fällen auch die supraorbitalen Ampullengruppen verwender (Abb. 1), da 
sich die von HENSEL (1955) und SAND (1938) untersuchte mandibuläre Ampullengruppe wegen der Kürze der zu den einzelnen Ampullen ziehenden afferenten Nerven nicht zur Ableitung der Aktivität von Einzelampullen eignet.

Nach Zerstörung des zentralen Nervensystems wurde die Ampullengruppe aus dem umgebenden Bindegewebe gelöst und mit Selachier-Ringer-Lösung bedeckt. Dann wurden die einzelnen Ampullen mit dem zugehörigen Nervenende von 2 bis $4 \mathrm{~mm}$ Länge vorsichtig herauspräpariert und in eine feuchte Kammer verbracht, deren Boden eine vergoldete Metallthermode bildete, die mit Wasser von verschiedenen Temperaturen durchströmt werden konnte. Dabei wurde darauf geachtet, daß die Ampullen stets mit einem dünnen Film von Selachier-Ringer-Lösung bedeckt waren. Die Ableitung der Impulse erfolgte mit Watte- oder bipolaren Platin-Elektroden vom ganzen Nerven, der aus 6 bis 8 Fasern besteht, oder von dünnen Nervenfilamenten. Nach Verstärkung mit einem Differentialvorverstärker wurden die Aktionspotentiale auf einen Tektronix-Kathodenstrahl-Oszillographen übertragen und gleichzeitig im Lautsprecher hörbar gemacht. Zur Temperaturregistrierung der Thermodeninnentemperatur diente ein NTC-Element, dessen Temperaturgang auf dem zweiten Kanal des Oszillographen zusammen mit den afferenten Impulsen photographisch aufgezeichnet wurde, während die Temperatur an der Thermodenoberfläche mit Thermoelementen des elektrischen Universalthermometers „Ellab“ kontrolliert wurde.

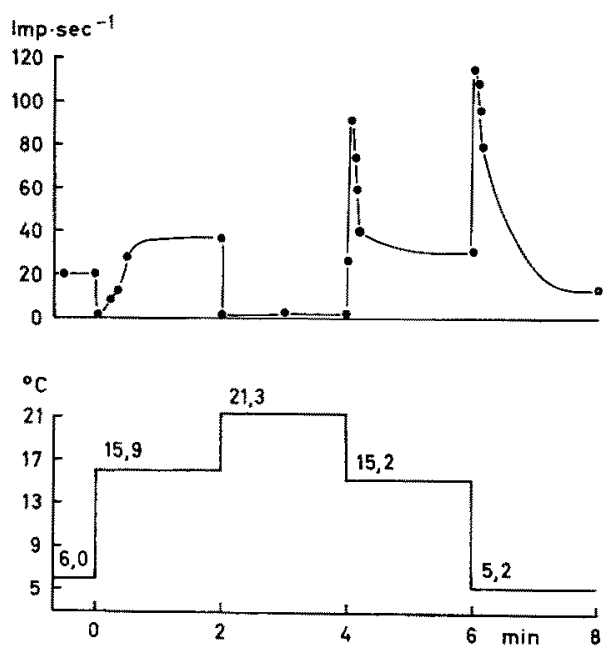

Abb. 2: Dynamische Impulsantwort einer Lorenzinischen Ampulle (infraorbitale Gruppe), die sich wie ein Kaltrezeptor verhält, auf plötzliche Temperaturerniedrigungen; Ordinate: oben Impulsfrequenz, unten Thermodentemperatur; Abszisse: Zeit in Minuten

\section{ERGEBNISSE}

Das Verhalten einer einzelnen Ampulle, deren Entladungsmuster dem eines Kaltrezeptors entspricht, ist auf Abbildung 2 wiedergegeben. Bei einer konstanten Temperatur von $6^{\circ} \mathrm{C}$ feuert die Ampulle mit einer Frequenz von 20 Impulsen pro sec. 
Während rascher Erwärmung auf $15,9^{\circ} \mathrm{C}$ tritt für wenige Sekunden eine vollständige Hemmung ein; dann steigt die Impulszahl wieder an und stellt sich auf einen konstanten Wert von 36 Impulsen/sec ein. Als Folge weiterer schneller Erwärmung auf
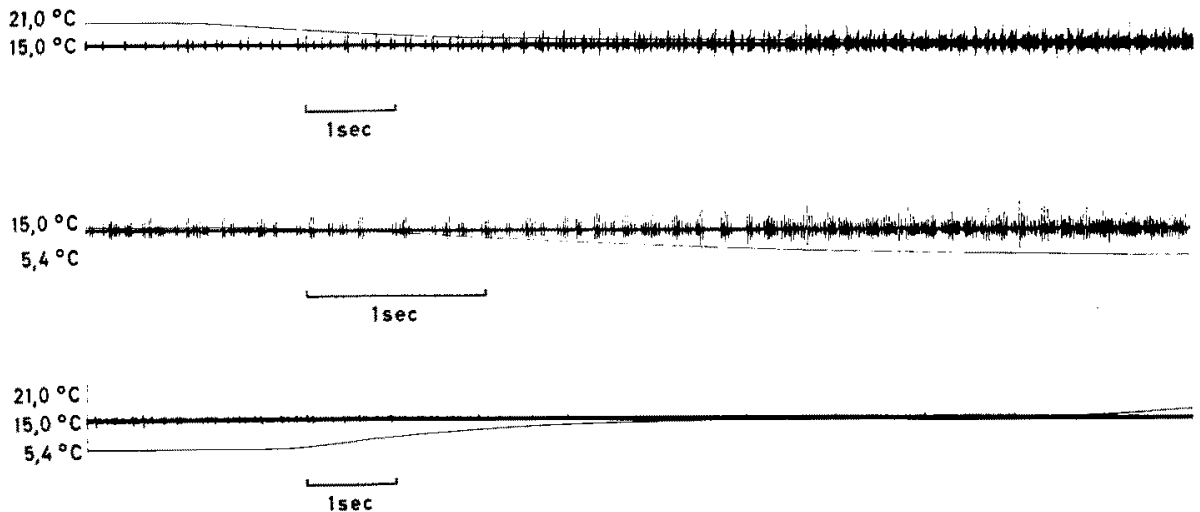

Abb. 3: Aktionspotentiale des afferenten Nerven einer isolierten Lorenzinischen Ampulle ("Kaltrezeptor") aus der infraorbitalen Ampullengruppe: oben und Mitte Abkühlung, unten Erwärmung; der zweite Strahl des Oszilloskops gibt die Thermodentemperatur wieder, deren Wert vor und nach Beendigung der jeweiligen Temperaturänderung auf der linken Seite angegeben ist

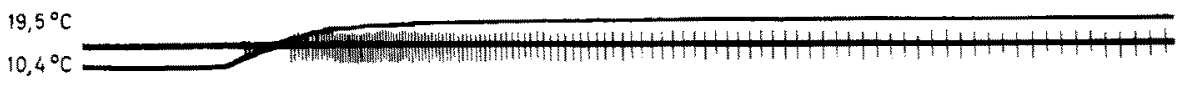

$19,5^{\circ} \mathrm{C}$
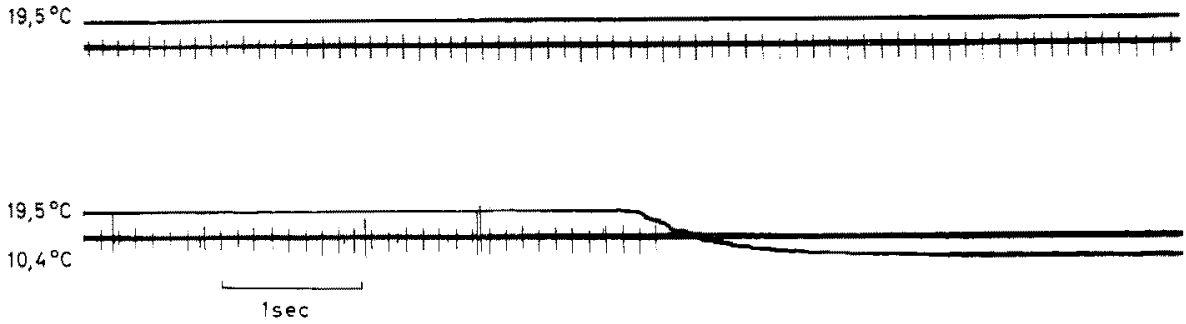

Abb. 4: Aktionspotentiale eines dünnen Filaments einer isolierten Lorenzinischen Ampulle (infraorbitale Gruppe), deren Frequenzmuster dem eines Warmrezeptors entspricht; Thermodentemperatur auf dem zweiten Strahl des Oszilloskops; oben: Erwärmung; Mitte: konstante Temperatur; unten: Abkühlung auf den Ausgangswert

$21,3^{\circ} \mathrm{C}$ werden die Spikes wieder vollständig gehemmt. Das Maximum der Entladungen bei stationären Temperaturen ist jetzt überschritten, so daß nach einer Minute die Impulse nur noch mit einer Frequenz von 2 bis 3 pro sec einsetzen. Beim Kältesprung wird die Impulsfrequenz vorübergehend stark erhöht, um sich dann innerhalb weniger Minuten auf einen neuen konstanten Wert einzustellen.

Die dynamische Antwort dieser Lorenzinischen Ampulle auf eine plötzliche Abkïhlung wird wie bei anderen Kaltrezeptoren von 3 Faktoren bestimmt: (1) Von der 
Höhe des Temperatursprunges, (2) von der Steilheit der Abkühlung und (3) von der Höhe der stationären Frequenzantwort bei der entsprechenden Ausgangstemperatur.

Abbildung 3 zeigt die Aktionspotentiale des afferenten Nerven einer solchen isolierten Lorenzinischen Ampulle: Bei Abkühlung jeweils überschießende Erregung, bei Erwärmung Hemmung der afferenten Entladungen. Der zweite Strahl des Oszillographen gibt die Thermodentemperatur wieder.

Die Impulse aus einem dünen Filament des afferenten Nerven einer anderen isolierten Ampulle sind auf Abbildung 4 dargestellt. Es fällt sofort auf, daß im Gegensatz zu den eben gezeigten Registrierungen bei schneller Erwärmung keine Hemmung, sondern Erregung eintritt. Schon nach $2 \mathrm{sec}$ wird die Impulsfolge langsamer, und nach $10 \mathrm{sec}$ ist ein konstanter Wert von 9 Impulsen/sec erreicht. Etwas später beginnt ein weiterer Rezeptor mit asynchronen Entladungen. Wird der gleiche Temperatursprung in umgekehrter Richtung vorgenommen, so kommt es zur vollständigen

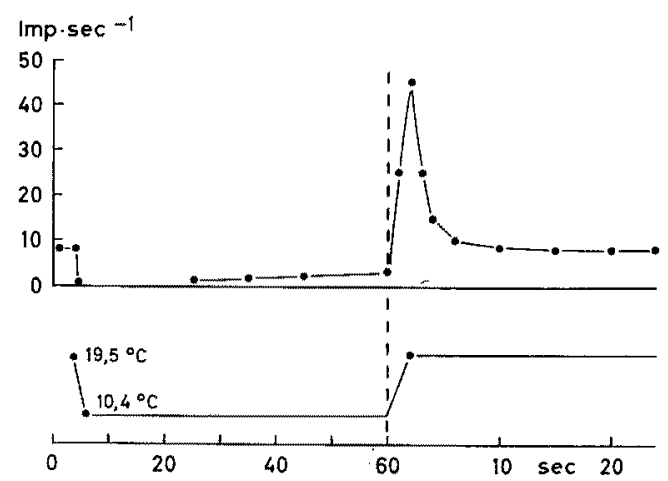

Abb. 5: Impulsantwort eines "Warmrezeptors“ als Mittelwertskurve aus 4 Kühl- und Wärmeperioden. Gleicher Rezeptor wie in Abbildung 4; Ordinate: Impulsfrequenz; Abszisse: Zeit in Sekunden; der Zeitabstand auf der Abszisse ist rechts von der gestrichelten Linie verdoppelt worden

Hemmung der Entladungen. Erst nach etwa $30 \mathrm{sec}$ beginnt der Rezeptor wieder mit einzelnen Spikes zu feuern (auf der Abbildung nicht dargestellt). Dieser Rezeptor verhält sich also gerade umgekehrt wie der in Abbildung 3 gezeigte. In seiner dynamischen Antwort auf Temperatursprünge entspricht er nach der Definition von HeNsel (1952, 1953) einem Warmrezeptor, dessen Frequenzmuster DODT \& ZOTTERMAN (1952) für den Warmbliter beschrieben haben.

Die Impulsantwort des gleichen Rezeptors auf einen Kälte- und Wärmesprung ist in Abbildung 5 als Mittelwertskurve aus vier Versuchen mit gleichen Temperatursprüngen abgebildet. Bei einer konstanten Temperatur von $19,5^{\circ} \mathrm{C}$ entlädt der Rezeptor mit 8 Impulsen/sec. Beim Kältesprung tritt eine vollständige Hemmung ein, nach $20 \mathrm{sec}$ beginnt der Rezeptor wieder zu feuern. Rasche Erhöhung der Temperatur auf den Ausgangswert führt zu einer durchschnittlichen Frequenzerhöhung auf 45 Impulse/sec, nach $10 \mathrm{sec}$ ist die ursprüngliche Impulszahl bei $19,5^{\circ} \mathrm{C}$ wieder erreicht. Das gleiche Frequenzverhalten eines anderen Rezeptors bei zwei Wärmesprüngen ist in Abbildung 6 wiedergegeben. Bei rascher Erwärmung von $10,5^{\circ}$ auf $20^{\circ} \mathrm{C}$ steigt die Im- 
pulszahl von 1 bis $2 / \sec$ auf 28 an, fällt dann wieder ab und stellt sich nach Durchlaufen eines Minimums auf einen neuen stationären Wert von $14 \mathrm{Impulsen} / \mathrm{sec}$ ein. Eine weitere Erwärmung auf $26,6^{\circ} \mathrm{C}$ führt zu einem vorübergehenden Frequenz-

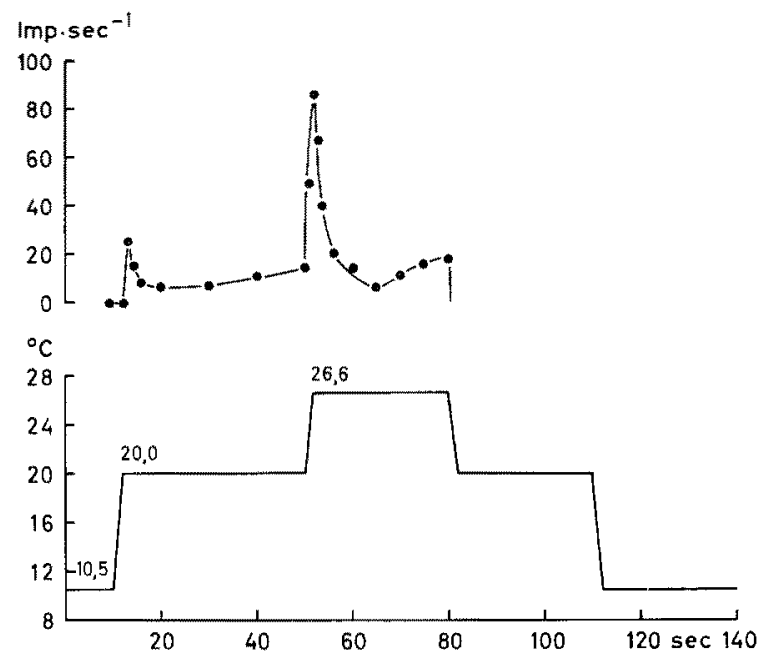

Abb. 6: Impulsantwort einer isolierten Lorenzinischen Ampulle, die sidh wie ein Warmrezeptor verhält, bei zwei Temperatursprüngen; Ordinate: oben Impulsfrequenz, unten Thermodentemperatur; Abszisse: Zeit in Sekunden

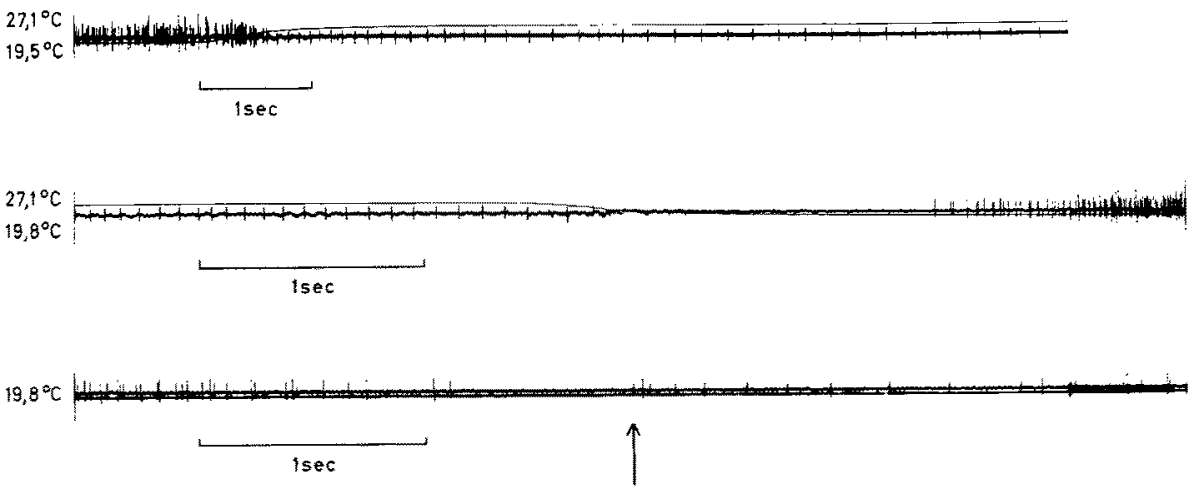

Abb. 7: Gesamtentladungen aus dem afferenten Nerven einer isolierten Lorenzinischen Ampulle bei schnellen Temperaturänderungen; o b e $n:$ bei Erwärmung werden die meisten Rezeptoren gehemmt; nur ein Rezeptor erhöht seine Entladungszahl; $\mathrm{M}$ it te : bei Abkühlung wird der durch Erwärmung aktivierte Rezeptor gehemmt, dafür beginnen die übrigen Anteile der Ampulle wieder zu feuern; u n $\mathrm{t}$ n : der gehemmte, Warmrezeptor" beginnt wieder mit langsamerer Frequenz zu feuern $(\uparrow)$.

anstieg auf 90 Impulse/sec, obgleich der Temperatursprung nur $\% / 3$ von der Höhe des vorhergehenden beträgt. Hier, zwischen $20^{\circ}$ und $26^{\circ} \mathrm{C}$, dürfte daher etwa das Maximum der stationären Antwortkurve liegen, also etwa $6^{0}$ bis $8^{0} \mathrm{C}$ höher als bei den Ampullen, die wie Kaltrezeptoren reagieren. 
Abbildung 7 zeigt die Gesamtentladungen eines afferenten Nerven einer einzelnen Ampulle des gleichen Tieres. Bei einer konstanten Temperatur von $19,5^{\circ} \mathrm{C}$ entlädt die Ampulle mit $58 \mathrm{Impulsen} / \mathrm{sec}$. Durch rasche Erwärmung auf $27,1^{\circ} \mathrm{C}$ werden die Rezeptoren bis auf einen gehemmt. Dieser erhöht seine Frequenz von ca. 3 bis 4 auf $15 \mathrm{Impulse} / \mathrm{sec}$, wird dann wieder langsamer und stellt sich auf eine stationäre Frequenz von 11 Impulsen/sec ein. Bei Abkühlung auf die Ausgangstemperatur wird er vorübergehend vollständig gehemmt. Dafür antworten die übrigen Fasern mit überschießender Erregung auf die Abkühlung. Nach $15 \mathrm{sec}$ beginnt auch der bei der Ab-

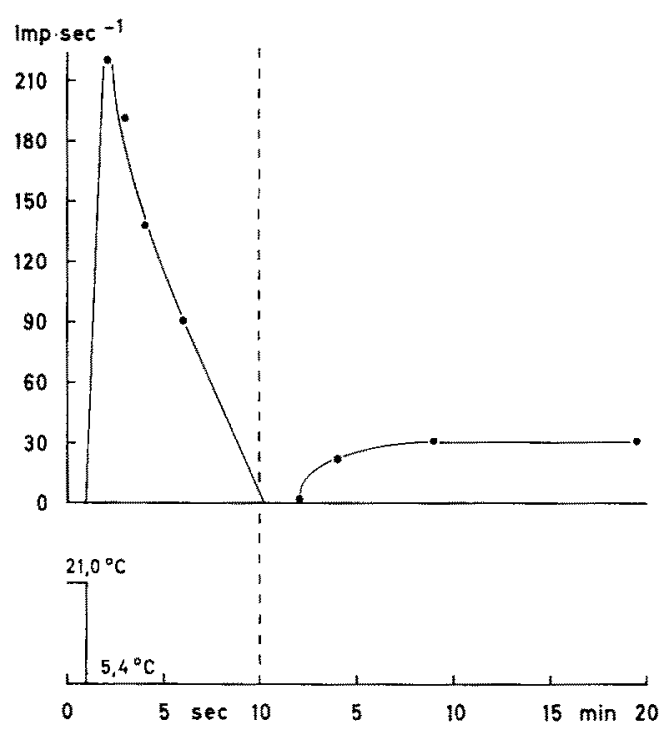

Abb. 8: Adaptives Verhalten einer isolierten Lorenzinischen Ampulle (infraorbitale Gruppe) nach plötzlicher Abkühlung; Ordinate: Impulsfrequenz; Abszisse: Zeit, links der gestrichelten Linie in Sekunden, rechts der gestrichelten Linie in Minuten

kühlung gehemmte Rezeptor wieder zu feuern. In dieser Ampulle befand sich also ein Rezeptor, der sich wie ein Warmrezeptor verhielt, während die übrigen Anteile der Ampulle gegenüber Temperaturänderungen das typische Verhalten von Kaltrezeptoren zeigten.

Anscheinend haben nur einzelne Rezeptoren in den Ampullen dieses umgekehrte Verhalten bei Temperatursprüngen, was ihr Auffinden wesentlich erschweren dürtte. Andererseits scheint das Vorkommen der "Warmrezeptoren" auf bestimmte Ampullengruppen beschränkt zu sein. Wir fanden sie nur in einigen infraorbitalen Ampullen von zwei Katzenhaien, während eine ganze Reihe von anderen infraorbitalen sowie alle untersuchten supraorbitalen Ampullen der gleichen Tiere sich bei einem Temperaturreiz ausschließlich wie Kaltrezeptoren verhielten. In den Einzelfaserpräparationen des afferenten Nerven der mandibulären Ampullengruppe haben weder HENSEL noch Murray einen Rezeptor gefunden, der in der Art eines Warmrezeptors reagierte.

Außer der Auffindung solcher "Warmrezeptoren" konnten wir in unseren Ver- 
suchen eine andere interessante Beobachtung machen. In den Einzelfaserpräparationen mandibulärer Ampullen ist bis auf einen Befund von Murray immer ein gleichmäßiges Abfallen der Impulszahl nach überschießender Erregung bzw. ein gleichmäßiger Anstieg nach Hemmung beschrieben worden. An einer großen Zahl von Ampullen konnten wir das gleiche Verhalten beobachten (Abb. 1). Eine Reihe von Ampullen zeigte aber ein anderes adaptives Verhalten nach Temperatursprüngen, wofür Abbildung 8 ein Beispiel gibt. Auf einen großen Temperaturabfall von $15^{\circ} \mathrm{C}$ reagiert die Ampulle

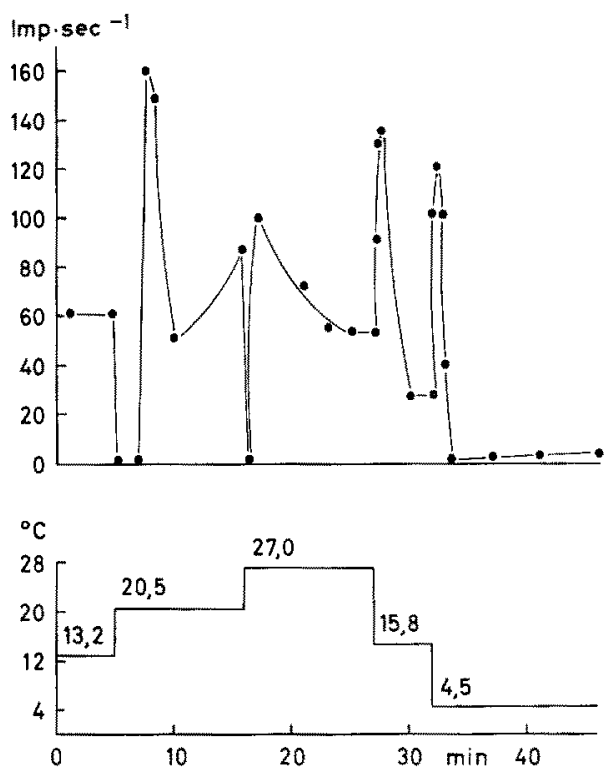

Abb. 9: Adaptationsverhalten einer isolierten Lorenzinischen Ampulle (infraorbitale Gruppe) nach Wärme- und Kältesprüngen; Ordinate: oben Impulsfrequenz; unten Thermodentemperatur; Abszisse: Zeit in Minuten

mit einer überschießenden Erregung. Nach $10 \mathrm{sec}$ sind die Impulse jedoch vollkommen erloschen. $Z_{\text {wei }}$ Minuten später zeigen sich wieder vereinzelte Aktionspotentiale, und nach etwa acht Minuten hat sich eine stationäre Frequenz eingestellt. Dieses Verhalten ist von der Größe des Temperatursprunges unabhängig. Die Adaptationszeiten waren bei den einzelnen Ampullen recht verschieden. Sie betrugen meist mehrere Minuten, bei einigen Ampullen aber auch nur Sekunden, wie zum Beispiel in Abbildung 7.

Diese Art der Adaptation tritt auch bei Erwärmung einer Reihe von Lorenzinischen Ampullen ein, die wie Kaltrezeptoren entladen (Abb. 9). Nach dem Wärmesprung von $13,2^{\circ}$ auf $20,5^{\circ} \mathrm{C}$ werden die Impulse für etwa 2 min gehemmt. Dann aber erfolgt nicht ein kontinuierliches Ansteigen der Impulszahl auf einen neuen stationären Wert, sondern es kommt ebenfalls zu einer überschießenden Erregung, der ein erneutes Absinken der Entladungsfrequenz folgt. Dann nimmt die Zahl der Spikes wieder zu. Beim Wärmesprung von $20,5^{\circ}$ auf $27^{\circ} \mathrm{C}$ ist es ganz ähnlich: Hemmung der Impulse, ïberschießende Erregung, dann aber im Unterschied zu vorher ein kontinuierlicher 
Abfall zu einer neuen stationären Entladungszahl. Die Adaptation bei schnellen Abkühlungen gleicht der vorher besprochenen. Dieses Verhalten isolierter Lorenzinischer Ampullen nach Temperaturänderungen fanden wir bei etwa der Hältte der Fälle, während in den anderen Versuchen die Einstellung auf stationäre Impulsfrequenzen nach der überschießenden Erregung beziehungsweise Hemmung kontinuierlich erfolgte.

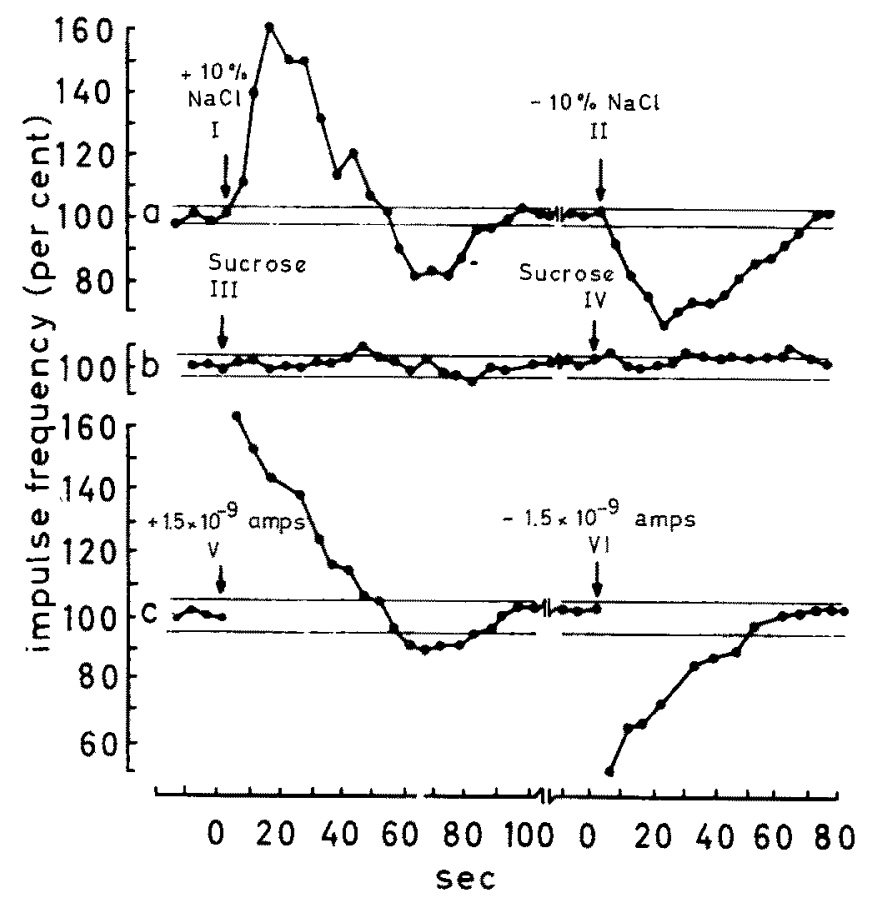

Abb. 10: Adaptationsverhalten isolierter Lorenzinischer Ampullen bei Anderung der $\mathrm{NaCl}-$ Konzentration (a) und bei elektrischer Reizung (c). (Nach LoEwENsTeIN \& IsHiko 1962)

Interessanterweise haben LoEwENSTEIN \& IsHiкo (1962) ein ähnliches Adaptationsverhalten isolierter Lorenzinischer Ampullen bei Anderung der Salzkonzentration beziehungsweise bei elektrischer Reizung festgestellt (Abb. 10). Wurde in dem an der Ampullenöfnung vorbeifließenden Meerwasser der NaCl-Gehalt um 10 Prozent erhöht, so stieg die Impulsfrequenz stark an, fiel dann aber unter den Ausgangswert, um schließlich auf einem neuen stationären Wert $z u$ adaptieren. Wie bei unseren Versuchen wurden von LoEWENSTEIN \& IsHiko infraorbitale Ampullen verwendet. MurRay (1959) fand unter Hunderten von mandibulären Ampullen eine, deren Frequenzgang dem oben beschriebenen gleicht. Außerdem fand er noch einige andere Ampullen, die vor dem Anstieg der Impulszahl bei Abkühlung einen kurzen Abfall und bei Erwärmung ein umgekehrtes Verhalten zeigten.

Daraus geht hervor, daß bei den infraorbitalen Ampullen der „abweichende Adaptationstyp" anscheinend viel häufiger ist als bei mandibulären Ampullen. Das gilt nicht nur für thermische, sondern auch für chemische und elektrische Reize. Da die 
Lorenzinischen Ampullen auf eine Reihe von Reizen ziemlich gleichartig antworten, könnte ein unterschiedlicher Adaptationstyp in den einzelnen Ampullengruppen zur Differenzierung des peripheren Reizmusters beitragen. Das würde bedeuten, daß die Lokalisation der Lorenzinischen Ampullen für ihre biologische Funktion von Bedeutung ist, wie Murray (1962) es vermutet. Für diese Ansicht spricht auch, daß „spezifische Leistungen", wie das Funktionieren einzelner Rezeptoren als "Warmrezeptor", anscheinend ebenfalls nur in bestimmten Ampullengruppen vorkommen.

\section{ZUSAMMENFASSUNG}

1. Es gibt in den Lorenzinischen Ampullen Rezeptoren, die sich bei raschen Temperaturänderungen wie Warmrezeptoren verhalten.

2. Eine Reihe von Lorenzinischen Ampullen zeigt nach Temperatursprüngen ein diskontinuierliches Adaptationsverhalten, das der Reaktion bei anderen Reizformen an isolierten Ampullen der infraorbitalen Gruppe sehr ähnlich ist.

3. Die Befunde legen den Schluß nahe, daß die Lokalisation der Ampullen sowohl für „spezifische" Rezeptorleistungen als auch für die Differenzierung des peripheren Reizmusters von Bedeutung ist.

Den Mitarbeitern der Meeresstation der Biologischen Anstalt Helgoland danken wir herzlich für freundliche Hilfsbereitschaft und Unterstützung.

\section{LITERATUR}

DijkgRaAf, S. \& Kalmuj, A. J., 1963. Untersudungen über die Funktion der Lorenzinischen Ampullen an Haifischen. Z. vergl. Physiol. 47, 438-456.

Dodt, E. \& Zotrerman, Y., 1952. Mode of action of warm receptors. Acta physiol. scand. 26, 345-357.

Hensel, H., 1952. Physiologie der Thermoreception. Ergebn. Physiol. 47, 166-368.

- 1953. Das Verhalten der Thermoreceptoren bei Temperatursprüngen. Pflïgers Arch. ges. Physiol. 256, 470 .

- 1955. Quantitative Beziehungen zwischen Temperaturreiz und Aktionspotentialen dex Lorenzinischen Ampullen. Z. vergl. Physiol. 37, 509-526.

- 1957. Die Wirkung verschiedener Kohlensäure- und Sauerstoffspannungen auf isolierte Lorenzinische Ampullen von Selachiern. Pfiügers Arch. ges. Physiol. 264, 228-244.

LoEWenstern, W. R., 1960. Mechanism of nerve impulse initiation in a pressure receptor (Lorenzinian ampulla). Nature, Lond. 188, 1034-1035.

- \& Ismko, N., 1962. Sodium chloride sensitivity and electrochemical effects in a Lorenzinian ampulla. Nature, Lond. 194, 292-294.

Murray, R. W., 1959. The response of the ampullae of Lorenzini to combined stimulation by temperature change and weak direct currents. J. Physiol., Lond. 145, 1-13.

- 1960. The response of the ampullae of Lotenzini of Elasmobranchs to mechanical stimulalation. J. exp. Biol. 37, 417-424.

- 1962. The response of the ampullae of Lorenzini of Elasmobranchs to electrical stimulation. J. exp. Biol. 39, 119-128.

SAND, A., 1938. The function of the ampullae of Lorenzini with some observations on the effect of temperature on sensory rhythms. Proc. R. Soc. (Ser. B) 125, 524-553. 


\section{Diskussion im Anschluß an den Vortrag Pierau \& MeIssner}

Precht: Auf Grund von Verhaltensversuchen kam Dijkgraf zu der Auffassung, daß die Lorenzinischen Ampullen als Elektrorezeptoren anzusehen sind.

PIeRau: Als spezifische 'Thermorezeptoren darf man die Lorenzinischen Ampullen wohl nicht ansehen, obwohl wir die Temperaturempfindlichkeit der Ampullen berïcksichtigen müssen. Die Versuche von Dijkgraf haben eine Klärung der biologischen Funktion noch nicht erbracht.

ZERBsT: Die Temperatur als "physiologische Reizskala" tritt wohl mehr oder weniger stark bei allen Rezeptoren der Kalt- und Warmblüter in Erscheinung, so bewirkt eine Abkühlung der Streckrezeptoren des Flußkrebses eine vorübergehende Frequenzzunahme - wie eine Dehnung. Aus Untersuchungen am rezeptorischen "Eingangsglied" sind wohl kaum Aussagen zur Temperaturspezifität möglich. BURKhardT (Biol. Zbl. 78, 22, 1959) zeigte, daß die Temperatur Vorgänge an der Rezeptorzelle und an der Schwelle für fortgeleitete Potentiale ge genläufig zu verändern vermag. So wird durch den Einfluß der Temperatur auf verschiedene Glieder des Informationskanals zum Beispiel eine Temperaturspezifiät ausgeschaltet oder "gepuffert". Untersuchungen an isolierten Rezeptorsystemen lassen daher keine Rückschlüsse darüber zu, ob tatsächlich Informationen über "Temperaturreize" zentral ankommen beziehungsweise "verarbeitet" werden.

Pifrau: Es gibt eine Reihe von Rezeptoren (Mechanorezeptoren, Muskelspindeln), die ein ähnliches Verhalten zeigen. Die Empfindlichkeit ist aber weit geringer, weshalb diese, wie im Fall der Muskelspindeln bei Warmblitern, unter physiologischen Bedingungen als Thermorezeptoren nicht in Betracht kommen. Da die Lorenzinischen Ampullen Temperaturänderungen gegenüber noch empfindlicher sind als die Thermorezeptoren der Warmblüter, deren Spezifität für zentrale Neurone LandgreEN (Acta physiol. scand. 48, 255, 1960) nachgewiesen hat, ist analog dazu die zentrale Informationsverarbeitung der auf die Lorenzinischen Ampullen wirkenden Temperaturreize durchaus denkbar. 